\title{
Advanced automated gain adjustments for in-vivo ultrasound imaging
}

Moshavegh, Ramin; Hemmsen, Martin Christian; Martins, Bo; Hansen, Kristoffer Lindskov; Ewertsen, Caroline; Brandt, Andreas Hjelm; Bechsgaard, Thor ; Bachmann Nielsen, Michael; Jensen, Jørgen Arendt

Published in:

Proceedings of IEEE International Ultrasonics Symposium

Link to article, DOI:

10.1109/ULTSYM.2015.0298

Publication date:

2015

Document Version

Peer reviewed version

Link back to DTU Orbit

Citation (APA):

Moshavegh, R., Hemmsen, M. C., Martins, B., Hansen, K. L., Ewertsen, C., Brandt, A. H., Bechsgaard, T., Bachmann Nielsen, M., \& Jensen, J. A. (2015). Advanced automated gain adjustments for in-vivo ultrasound imaging. In Proceedings of IEEE International Ultrasonics Symposium IEEE.

https://doi.org/10.1109/ULTSYM.2015.0298

\section{General rights}

Copyright and moral rights for the publications made accessible in the public portal are retained by the authors and/or other copyright owners and it is a condition of accessing publications that users recognise and abide by the legal requirements associated with these rights.

- Users may download and print one copy of any publication from the public portal for the purpose of private study or research.

- You may not further distribute the material or use it for any profit-making activity or commercial gain

- You may freely distribute the URL identifying the publication in the public portal 


\title{
Advanced Automated Gain Adjustments for In-Vivo Ultrasound Imaging
}

\author{
Ramin Moshavegh*, Martin Christian Hemmsen*, Bo Martins ${ }^{\ddagger}$, Kristoffer Lindskov Hansen ${ }^{\dagger}$, Caroline Ewertsen ${ }^{\dagger}$ \\ , Andreas Hjelm Brandt ${ }^{\dagger}$, Thor Bechsgaard ${ }^{\dagger}$, Michael Bachmann Nielsen ${ }^{\dagger}$ and Jørgen Arendt Jensen* \\ ${ }^{*}$ Center for Fast Ultrasound Imaging, Dept. of Elec. Eng., Technical University of Denmark, Lyngby, Denmark \\ †'Department of Radiology, Copenhagen University Hospital, Copenhagen, Denmark \\ ${ }_{\ddagger}^{\ddagger}$ BK Ultrasound ApS, Herlev, Denmark
}

\begin{abstract}
Automatic gain adjustments are necessary on the state-of-the-art ultrasound scanners to obtain optimal scan quality, while reducing the unnecessary user interactions with the scanner. However, when large anechoic regions exist in the scan plane, the sudden and drastic variation of attenuations in the scanned media complicates the gain compensation. This paper presents an advanced and automated gain adjustment method that precisely compensate for the gains on scans and dynamically adapts to the drastic attenuation variations between different media. The proposed algorithm makes use of several ultrasonic physical estimates such as scattering strength, focus gain, acoustic attenuation, and noise level to gain a more quantitative understanding of the scanned media and to provide an intuitive adjustment of gains on the scan. The proposed algorithm was applied to a set of 45 in-vivo movie sequences each containing 50 frames. The scans are acquired using a recently commercialized BK3000 ultrasound scanner (BK Ultrasound, Denmark). Matching pairs of in-vivo sequences, unprocessed and processed with the proposed method were visualized side by side and evaluated by 4 radiologists for image quality. Wilcoxon signed-rank test was then applied to the ratings provided by radiologists. The average VAS score was highly positive 12.16 ( $p$-value: $2.09 \times 10^{-23}$ ) favoring the gain-adjusted scans with the proposed algorithm.
\end{abstract}

\section{INTRODUCTION}

In ultrasound imaging the scanned media strongly attenuate the RF signals and therefore, they span a wide dynamic range when they are received by the transducer. Time gain compensation (TGC) is usually used to compensate for the acoustic attenuation before received echoes are visualised on the scanner. TGC offsets the attenuation of the RF signals along the depth, so those echoes belonging to the deep structures are more amplified compared to the echoes from superficial structures. The result is a more uniform image to be displayed on the scanner [1]. A simple curve is usually used in the current scanners for the TGC. The user manually adjusts the shape of this curve using sliders corresponding to different depths. However, the image quality is highly dependent on the adjustments, and they are rarely used by the users. An automatic adjustment of the gains (ATGC) without user intervention obviates the shortcomings of the manual TGC [2]. However, few designs are proposed in the literature [1]-[3]. Despite their advantage of reducing the interaction, such methods have an inherent draw-back. These methods consider that large and homogeneous media exist while scanning, and as a consequence, the dependency of the acoustic attenuation in the presence of different tissues and structures is not highlighted. Therefore, they fail to adjust the TGC and overall gain when large fluid collections such as urine bladder or gallbladder exist [1], [3], [4]. To address the issue, a solution that integrates several measures based on a physical understanding of the scanned media must be implemented.

The objective of this paper is to propose an advanced automated gain adjustment algorithm based on the scattering strength, signal-to-noise ratio, focusing gain, and the acoustic attenuation. From these parameters, different structures in the scanned media are determined automatically, and gains are precisely adjusted in the scanned media. The remainder of this paper is organized as follows. Section 2 introduces the proposed algorithm. Section 3 presents the measurement setups of the proposed algorithm and discusses the findings. Finally Section 4 gives the discussion and conclusion.

\section{Advanced Automated Gain Adjustments}

The Advanced Automated Gain Adjustment (ADGA) contains five distinct steps. The steps are discussed accordingly in the following sections.

\section{A. Insonified-region detection}

The purpose of the first step was to determine the insonifiedregion on the ultrasound scans. Long and dark shadows, starting from the top and extending to edge of the display, sometimes appear on one or both sides of ultrasound scans. This is usually when the transducer is not perfectly attached to the surface during a normal scan session. Therefore, detection of insonified region and removing the dark shadows from the gain adjustment process is necessary. For this purpose, the envelope of the RF data was computed. A binary mask, only including the intensity values lying within lower $10 \%$ dynamic range of the envelope data was generated . B-lines longer than $90 \%$ of the total scan depth were kept on that binary mask and shorter B-lines were excluded. Objects that were not attached to one of the right or left borders were also excluded from the mask. This made sure that only elongated and very dark regions on one or both sides of the scan remained. Those regions were considered as areas that were not insonified and were excluded from the gain adjustments. 


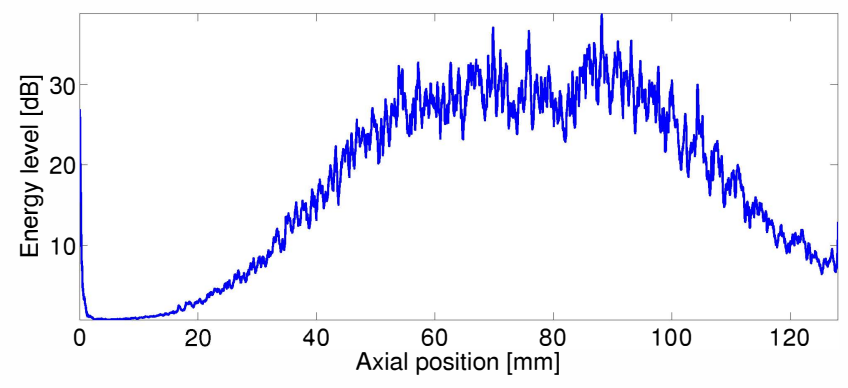

(a) Median of the energy levels of 50 scan lines.

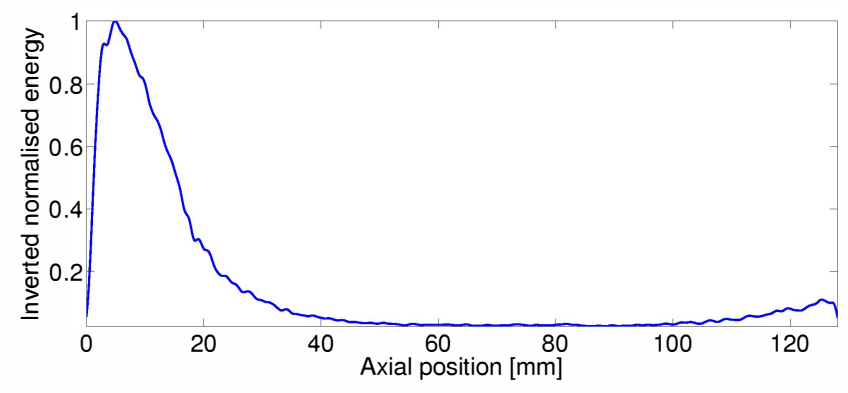

(b) Inverted and normalized energy curve in $(a)$.

Figure 1. Energy equalization step.

\section{B. Energy equalization}

The main purpose of the second step was to make sure that the energy is equally distributed throughout the scanned media and its evenly highlighted. An experiment was conducted in which a tissue mimicking speckle phantom was scanned, and 50 frames were acquired. The mean of all 50 frames computed yielded a mean frame. The lateral median of the 50 scan lines bracketing the center line on the mean frame was computed yielded an energy curve as a function of depth. This is shown in Fig. 1a. The curve was then inverted, normalized to a maximum of one, and used to scale the energy levels of all the lines in the image (Fig. 1b).

\section{2-D SNR map computation}

2-D signal to noise ratio (SNR) maps of the acquired frames were computed from the complex IQ data with the method previously introduced by authors [5]. The noise power of the ultrasound device is first computed. Then, the SNR map for an acquired in-vivo ultrasound frame is computed by dividing the signal power of the frame by the noise power of the system. An example of the 2-D SNR map computed from scan of the human liver is shown in Fig. 2a. Fluid collections depict very low SNR values in the SNR maps. Two features are then used to characterize this property. First, the cumulative frequency histogram of all SNR values inside the SNR map is generated. Second, a curve is fitted to the histogram values. Third, the amount of fluid in the scan was then characterized by slopes of the curve at 25th and 80th percentiles (Fig. 2b).

\section{Dynamic TGC}

A TGC curve is dynamically computed for each frame and used to compensate the signal levels. For this purpose, the

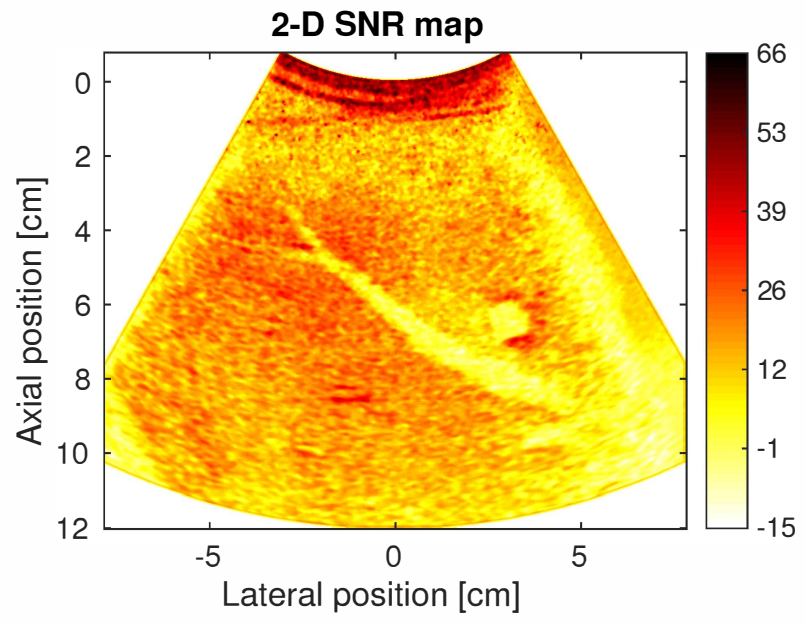

(a) 2-D SNR map computed for a scan of human liver.

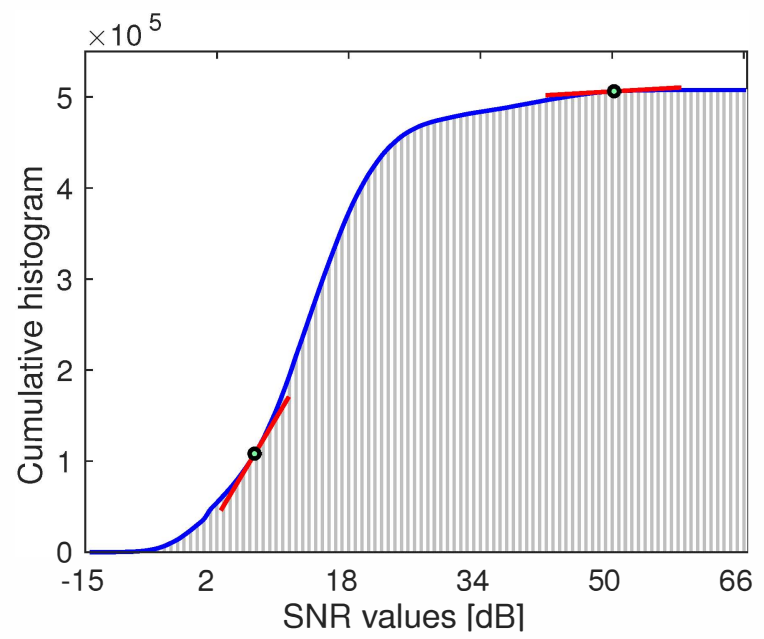

(b) Slope of the histogram at 25th (feature $f_{1}$ )and the 80th (feature $f_{2}$ ) percentiles.

Figure 2. 2-D SNR maps for a scan of human liver and the slope of histogram at 25 th (feature $f_{1}$ ) and the 80th (feature $f_{2}$ ) percentiles.

envelope of the acquired scan is first computed. A cumulative histogram of the intensity values is then generated. The intensity values less than half of the maximum intensities in the envelope are disregarded, and a mask of strong signal regions is generated. The mask is applied to the envelope, and the lateral median of the intensities lying inside the mask is computed. The computed curve presents drastic fluctuations and can not be used directly to analyse the axial intensity changes in the envelope. To address this, the curve is smoothed using a Sovitsky-Golay filter. Then the general trend of the curve is extracted by applying the singular-spectrum analysis (SSA). SSA does not require model of time series, and extracts the trend of time series in the presence of noise and oscillations [6]. The final curve depicts the trend of intensity change within the envelope and no sudden fluctuations (Fig. 3b). The curve is finally normalized to it's maximum, inverted, and applied as a TGC to the envelope to compensate for the gradual changes in the intensities. The dynamic adjustment of intensities using this curve might over-gain inside the fluid regions. Therefore, 


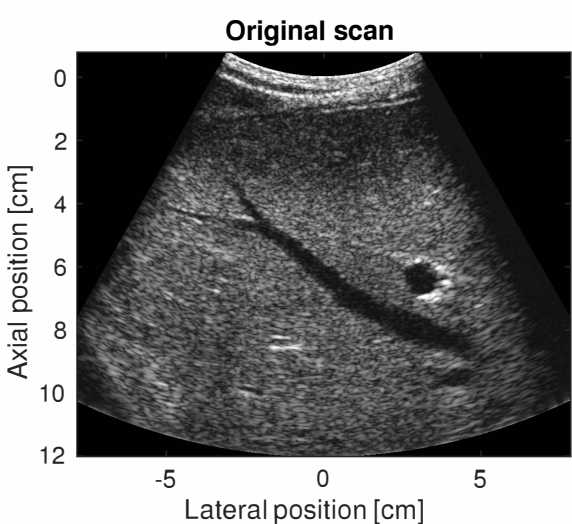

(a) Scan for which the TGC curve is computed. (b) TGC curve computed for $(a)$.

Figure 3. Illustration of a TGC curve dynamically computed for a frame of human liver scan.

the next step computes an attenuation map that can be used to correct the possible miss-adjusted gains inside the fluid regions after applying the TGC curve.

\section{E. 2-D attenuation map}

2-D attenuation maps of the acquired scans were determined by a spectral difference method adapted from Kuc [7]. The attenuation maps were computed from the beamformed RF data. RF lines were segmented to overlapping blocks. For each two overlapping blocks, slope of a line fitted to the power spectral difference of the two blocks was computed (Fig. 4). That slope was considered as the attenuation value for the top block. The overlapping blocks were moved on the RF data, several attenuation coefficients were then calculated, and finally a 2-D attenuation map was determined. The method is discussed in [5]. The attenuation map was normalized to a maximum of one and multiplied to the gain-adjusted frame by the dynamic TGC in Section II-D. This was performed to correct the gains inside the fluid collections.

\section{MEASUREMENT SETUP}

The proposed ADGA algorithm was applied to 45 in-vivo ultrasound sequences. Nine subjects were scanned, and a total of 45 sequences, each containing 50 frames were acquired. A collection of different anatomical locations such as urine bladder, gallbladder, kidney, liver, and carotid artery was acquired and included in the dataset. This was done to evaluate the performance of the ADGA on the scans including large fluid collections and those horboring less-variable attenuation distribution. The beamformed RF data were acquired using a recently commercialized BK3000 scanner (BK Ultrasound, Denmark) connected to a 192 -element $3.5 \mathrm{MHz}$ convex array transducer (9040, BK Ultrasound). The acquired sequences were generated by the dynamic receive focusing technique with factory preset TGC. The beamformed RF data were simultaneously recorded by a research interface on the scanner, and processed with the proposed algorithm off-line. Fig. 5 shows an in-vivo result of the algorithm applied to scans of a human gallbladder and inferior vena cava. To empirically evaluate the performance of the algorithm, matching Pairs of invivo sequences, unprocessed and processed with the proposed algorithm, were evaluated side by side by four experienced radiologists. The evaluation was double blinded, and each pair was shown twice by randomizing the left and right positioning. This resulted in a total of 360 independent visual evaluations. During the evaluation, each radiologist was asked to rate the image quality of the pairs on a visual analogue scale (VAS) ranging between -50 and +50 . The rating was performed by moving a slider towards their favored movie sequence. A positive scale favored the gain-adjusted sequence with the proposed algorithm. Wilcoxon signed-rank hypothesis test was applied to the provided ratings by the radiologists to check whether radiologists preferred the sequence with or without the proposed algorithm. Visualization and assessment were handled using the program IQap[8].

\section{Discussion AND CONCLUSION}

The average VAS score computed from the provided ratings was highly positive ( $p$-value: $2.09 \times 10^{-23}$ ) and estimated to be 12.16 favoring the gain-adjusted data with the proposed algorithm. This paper presented an advanced automated gain adjustment algorithm that utilises the estimates of attenuation, SNR, and focus gain to adjust the gains on the ultrasound scans. The proposed method is automatic, robust, and demonstrates reliable gain adjustments especially inside the large fluid collections. Also, the automatic adjustment of gains on the scans significantly reduces the unnecessary and manual gain adjustments on the ultrasound scanners. This highly increases the performance of the clinical staff during the scan session.

\section{ACKNOWLEDGMENT}

This work was financially supported by grant $82-2012-4$ from the Danish Advanced Technology Foundation and from BK Medical ApS, Herlev, Denmark.

\section{REFERENCES}

[1] D. Lee, Y. S. Kim, and J. B. Ra, "Automatic time gain compensation and dynamic range control in ultrasound imaging systems," Proc. SPIE Med. Imag., vol. 6147, pp. 614708-614708-9, 2006.

[2] S. Pye, S. Wild, and W. McDicken, "Adaptive time gain compensation for ultrasonic imaging," Ultrasound Med. Biol., vol. 18, pp. 205-212, 1992.

[3] M. Tang, F. Luo, and D. Liu, "Automatic time gain compensation in ultrasound imaging system," in Bioinformatics and Biomedical Engineering. ICBBE 2009.3rd International Conference on, June 2009, pp. 1-4.

[4] J. Litniewski, Z. Klimonda, and A. Nowicki, "Parametric sonographic imaging-application of synthetic aperture technique to imaging attenuation of ultrasound in tissue structures," Hydroacoustics, vol. 15, pp. 99-110, 2012.

[5] R. Moshavegh, M. C. Hemmsen, B. Martins, A. H. Brandt, K. L. Hansen, M. B. Nielsen, and J. A. Jensen, "Automated hierarchical time gain compensation for in-vivo ultrasound imaging," Proc. SPIE, vol. 9419, pp. 941 904-941 904-9, 2015.

[6] N. Golyandina, V. Nekrutkin, and A. A. Zhigljavsky, Analysis of time series structure: SSA and related techniques. CRC press, 2001.

[7] R. Kuc, "Estimating acoustic attenuation from reflected ultrasound signals: Comparison of spectral-shift and spectral-difference approaches," IEEE Trans. Acous., Speech, Sig. Pro., vol. 32, no. 1, pp. 1-6, 1984.

[8] M. C. Hemmsen, M. M. Petersen, S. I. Nikolov, M. B., Nielsen, and J. A. Jensen, "Ultrasound image quality assessment: A framework for evaluation of clinical image quality," in Proc. SPIE Med. Imag., vol. 76291 pp. $76290 \mathrm{C}-12,2010$. 

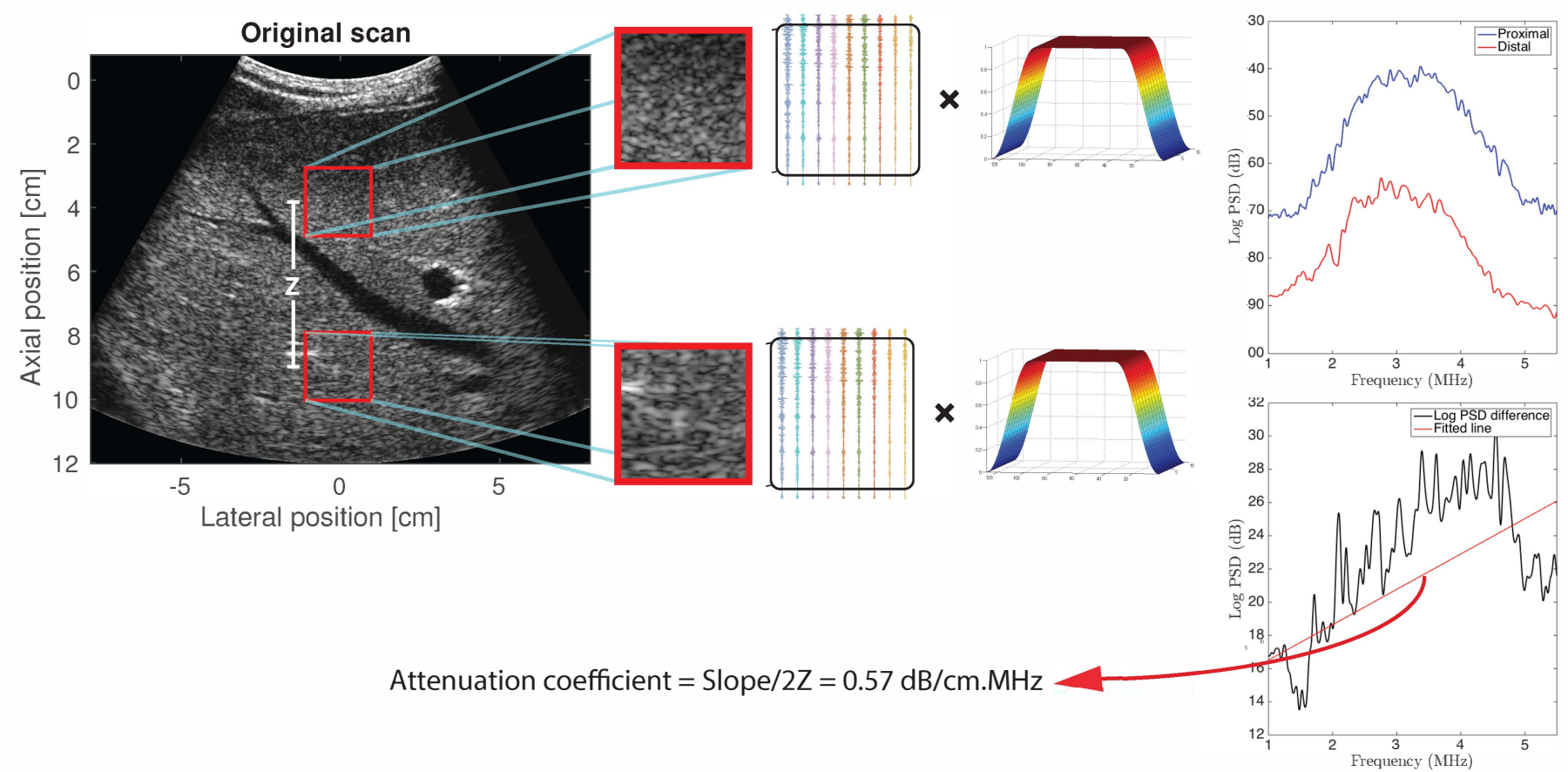

Figure 4. Illustration of how attenuation coefficients are computed.
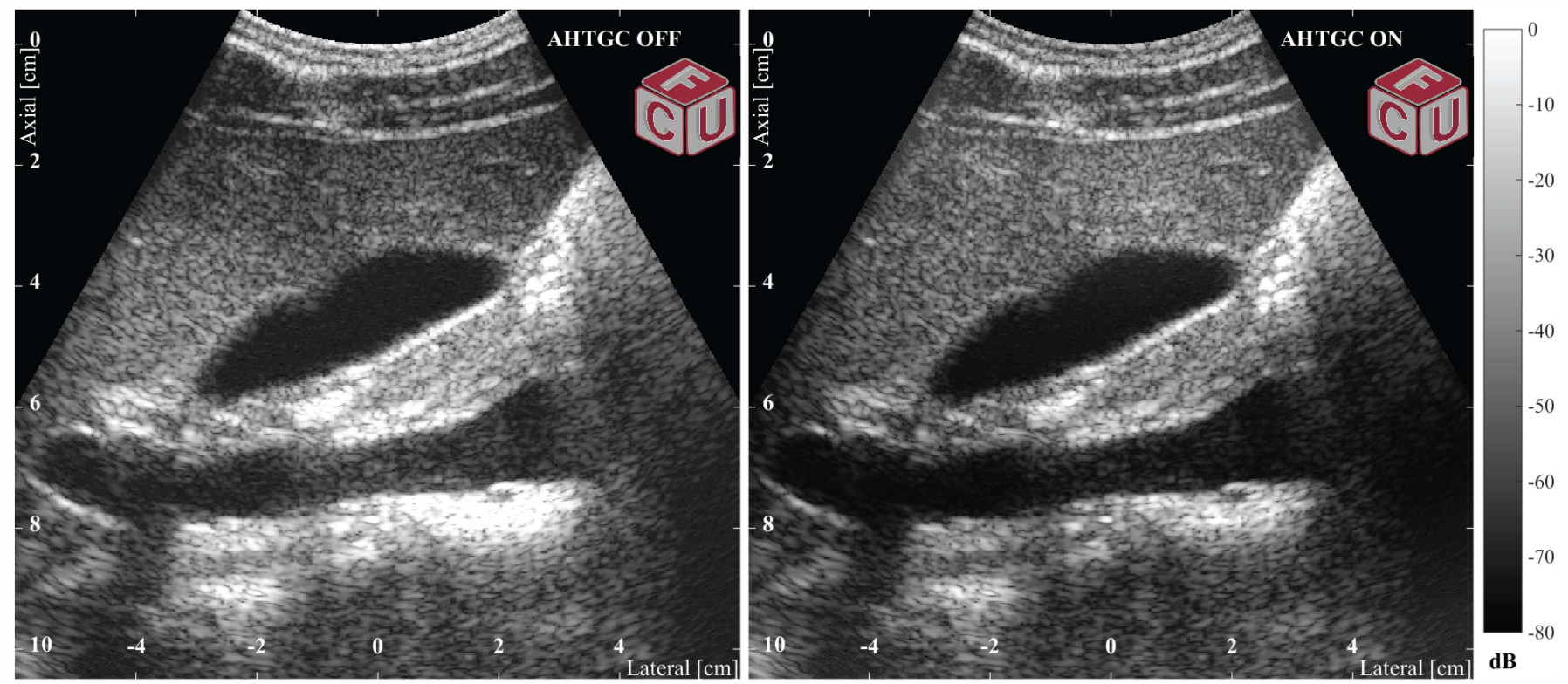

Figure 5. Sagittal view of a human gallbladder and inferior vena cava. 